\title{
Prognostic Value of Programed Cell Death-1 Ligand Expression in Colorectal Cancer and Its Correlation with Cytotoxic Tumor-Infiltrating Lymphocytes Ghada E. Esheba \\ Department of Pathology, Faculty of Medicine, Tanta University, Tanta, Egypt \\ * Corresponding author:Ghada E Esheba ,email: isia992018@yahoo.com
}

\begin{abstract}
Background: colorectal cancer (CRC) is a major cause of cancer-related death worldwide. In recent years, targeting the programed cell death (PD-1)/ programed cell death-1 ligand (PD-L1) immune checkpoint signaling has been tested as a novel promising treatment strategy in several tumors. Aim of the work: the present study aimed to examine expression of PD-L1 and CD 8+ T cells in CRC and to evaluate the relationship between their expressions and the different clinicopathological features. Material and methods: expression of PD-L1 in tumor cells (TC) as well as in tumor infiltrating immune cells (TIMC) was separately evaluated in 85 cases of CRC by the immunohistochemistry, in addition, CD8+ T cell count was assessed. Results: $12.9 \%$ of cases showed PD-L1 expression in TC while, $28.2 \%$ showed PD-L1expression in TIMC. PD-L1 expression in TC was significantly associated with higher tumor grade (P $=0.001)$, lymph node metastasis $(\mathrm{LNM})(\mathrm{P}=0.006)$ and lymphovascular invasion $(\mathrm{LVI})(\mathrm{P}<0.001)$. On the other hand, PDL-1 expression in TIMC was significantly associated with low tumor grade $(\mathrm{P}=0.016)$, negative LVI $(\mathrm{P}=$ 0.004), high tumor infiltrating lymphocytes (TILs) count $(\mathrm{P}<0.0001)$, as well as high CD8+ T cells count $(\mathrm{P}=0.002)$. High intra CD8-positive T cells was significantly associated with absence of LNM $(\mathrm{P}=0.013)$ and negative LVI $(\mathrm{P}$ $=0.035)$. Conclusion: these findings indicated that expression of PD-L1 can be used as a new biomarker to predict the prognosis of colon cancer and may provide a clue for immunotherapy in the adjuvant treatment of CRC patients.
\end{abstract} Keywords: colorectal cancer, PD1, PDL-1, CD8 + lymphocytes.

\section{INTRODUCTION}

Colorectal cancer is one of the most common cancers in men and women and is a major cause of cancer-related death worldwide. Although significant advances in multidisciplinary therapies have improved the outcome, the overall prognosis remains poor especially in advanced stages. Furthermore, treatment modalities used for advanced stage CRC have been found to be of modest potency and are associated with serious toxicities ${ }^{(1-3)}$. Recently, immunotherapy by using inhibitors against immune checkpoint is considered as a novel modality for the treatment of various malignancies ${ }^{(4)}$.Programmed death-1 (PD-1) receptor and its ligand, programmed death ligand-1 (PD-L1), have been reported as the major immune checkpoint targets for immunotherapy in many types of cancers such as melanomas, non-small-cell lung cancers, renal cell carcinomas, bladder cancers, ovarian carcinoma and breast carcinomas ${ }^{(5)}$.PD-L1, known also as $\mathrm{B} 7-\mathrm{H} 1$ or $\mathrm{CD} 274$ is a transmembrane glycoprotein encoded by the PD-L1 gene and is one of B7 family members. The latter have been reported to downregulate T-cell activation. PD-L1 is expressed by the immune cells such as $\mathrm{T}$ and $\mathrm{B}$ lymphocytes, macrophages and dendritic cells as well as nonimmune cells as endothelial, pancreatic and human tumors ${ }^{(6)}$.PD1, the receptor for PD-L1, is a member of the CD28 family and is expressed on activated T cells and can also be induced on activated $\mathrm{B}$ cells and natural killer cells ${ }^{(7)}$. PD-1/PD-L1 signaling inhibits CD8 cytotoxic immune reaction, $\mathrm{T}$ cell migration, proliferation and secretion of cytokine (such as IFN- $\gamma$ and IL-2) production, resulting in T lymphocyte anergy and/or apoptosis, induction of immunological tolerance and limiting cancer cell killing. Subsequently, the antitumor immune response is dramatically impaired. To switch back this unfavorable situation, several blockades of the PD-1/PD-L1 pathway have been developed ${ }^{(1)}$.

Theoretically, blocking of PD-1/PD-L1 signaling can restore the native anti-tumor function of $\mathrm{T}$ cells and facilitate tumor regression, therefore, PDL1 and PD-1 expressions have been addressed as predictive markers for tumor response to anti-PD-1 or PD-L1 immunotherapy in different malignancies and as potential targets for cancer therapy ${ }^{(4)}$.Tumorinfiltrating lymphocytes (TILs) mirror the host immune response directed against tumors. Furthermore, tumor infiltration by CD8+ cytotoxic T lymphocytes has been found to be associated with a good prognosis and an improved survival of colorectal cancer patients ${ }^{(6,8,}$ ${ }^{9}$.PD-L1 expression in tumor cells has been linked to a weak host immune response and eventual bad prognosis in several malignancies, including melanoma and esophageal, gastric, hepatocellular, non-small cell lung cancer and urothelial carcinomas, however, the prognostic significance of PD-L1 expression in colorectal cancer is still controversial whether its expression indicates a better or worse prognosis $(5,10,11)$. Also, a previous study showed that PD-L1 expression in tumor cells in gastrointestinal malignancies is less frequent compared to other solid tumors such as melanoma ${ }^{(12)}$. Therefore, this study was conducted to evaluate expression of PD-L1 in both tumor cells and in the tumor-infiltrating immune cells/cancer microenvironment (stroma) and to investigate the relationship between PD-L1 expression and the different clinico-pathological variables. Also, this study 
aimed to assess intra- and peritumoral/stromal CD8+ T cell density and correlate it with the clinico-pathological variables with PD-L1 expression.

\section{MATERIAL AND METHODS Material}

Eighty five cases of CRC were included in this study. Formalin-fixed, paraffin-embedded tissue blocks containing CRC were obtained retrospectively from the archives of the Department of Pathology, Faculty of Medicine, Tanta University during the period between March 2010 and March 2017. Clinical data of the CRCs were retrospectively collected by medical record review. The inclusion criteria included: all cases that were diagnosed as colon cancer provided that the clinical data such as age, tumor size, lymph node status and stage were present. Cases with no available data were excluded from this study. Evaluation of the histopathological features was performed. The histological parameters assessed were tumor size, tumor grade, histological subtype, mucinous histology, medullary histology, depth of invasion (pT), lymph node involvement/metastasis $(\mathrm{pN})$, extent of tumor infiltrating lymphocytes (TILs), lymphovascular and perineural invasions ${ }^{(13)}$.

This work has been approved by the local institutional ethical committee in accordance with the principles of the Declaration of Helsinki.

For the histopathological study, the sections were stained with hematoxylin and eosin.

\section{Methods}

\section{Immunohistochemistry}

Immunohistochemistry was performed on $4-\mu \mathrm{m}$ sections that were cut by routine paraffin embedded blocks using an automated stainer (Benchmark Ventana, Tucson, AZ) and the following antibodies according to the standard protocol and the manufacturer's recommendations for each antibody: PD-L1 (1:50, ab174838, Abcam, Cambridge, MA, USA), CD8 (clone: IgG2b 4B11, RTU, Leica, IL).

Normal placenta was used as an external positive control for PDL-1. Negative control was obtained by use of non-immune normal mouse serum as the primary antibody.

\section{Scoring system \\ Evaluation of PD-L1 expression in TCs}

PDL-1 was expressed on the cell membrane and also in the cytoplasm of the tumor cells. Membranous expression was scored as follow: $0=<5 \%$ of the tumor cells; $1=\geq 5 \%$ of tumor cells with membranous staining of any intensity. Cytoplasmic staining was scored as follow: 0 (negative), 1 (weak), 2 (moderate) and 3 (strong). A combined PD-L1 score was calculated by the sum of the membranous and cytoplasmic (intensity) scores, with a minimum score of zero and a maximum combined score of four. Scores of 0 and 1 were considered as negative, while scores of 2,3 , and 4 were considered as positive for PD-L1 expression ${ }^{(6)}$.

\section{Evaluation of PD-L1 expression in TIMCs}

PD-L1 expression on TIMCs was evaluated as follow: $0(<1 \%), 1(\geq 1$ and $<5 \%), 2(\geq 5 \%$ and $<10 \%)$, or 3 $(\geq 10 \%)$. Scores of 0 and 1 were considered as PD-L1 negative and scores of 2 and 3 were PD-L1 positive, as previously described ${ }^{(1)}$.

\section{Evaluation of TILs}

The extent of TILs in H\&E stained sections was semiquantitatively scored using a 4-degree scale as follow: 0 (absent), 1 (<30\%), $2(30 \%-60 \%)$ and $3(>60 \%)$. Tumors with a score of 0 or 1 were considered low while, tumors with a score of 2 or 3 were considered high ${ }^{(14)}$.

\section{Evaluation of CD8 positive $T$ cell numbers}

All biopsies were first scanned at low-power magnification $(10 \mathrm{x})$ and the five densest regions of intratumoral CD8+ (intra CD8, CD8 lymphocytes that infiltrate in between tumor cells) and peritumoral/stromal CD8+ (peri CD8, CD8 cells that infiltrate into the cancer stroma) were recognized. Within these areas, intra CD8 and peri CD8 T-cells were counted in 5 high-power fields each. The average number of infiltrating $\mathrm{T}$-cells per high power field was determined for each area.

\section{Scoring for CD8 positive $T$ cell numbers}

Intratumoral CD8-positive $\mathrm{T}$ cells were scored as low or high according to their mean number if $<50$ or $\geq 50$, respectively. Peritumoral CD8-positive $\mathrm{T}$ cell were scored as low or high according to their mean number if $<200$ or $\geq 200$, respectively. This scoring method was approved in colorectal cancer ${ }^{(10)}$.

\section{Statistical analysis}

Data analyses were carried out by the software of SPSS version 22 for Windows (SPSS Inc, IL, USA). Data were presented as median for continuous variables or percentages as appropriate. Associations between PDL-1 expression and CD8+ lymphocytes and the categorical clinicopathological variables were tested using Chi-square test. Fisher exact tests were used when appropriate. The $\mathrm{P}$ values were reported and the differences were considered statistically significant when $\mathrm{P}$ was less than 0.05 .

\section{RESULTS \\ Patient characteristics}

The median age was 64 years (range, 39-87 years) and $54 \%$ of the patients were male. A summary of clinicopathological characteristics is shown in table 1. 


\section{PD-L1 Expression in Tumor Cells}

Using 5\% cut-off, PD-L1 was expressed on the cell membranes and the cytoplasm of tumor cells in 11 cases (12.9\%) (Fig. 1B-F). Interestingly, PD-L1 expression was also detected in the normal mucosal glands (Fig. 1A).

As shown in table 2, positive PD-L1 expression in tumor cell was associated with histopathological features of aggressive tumor behavior such as higher tumor grade $(\mathrm{P}=0.001)$, presence of lymph node metastases $(\mathrm{P}=0.006)$, as well as the presence of lymphovascular invasion $(\mathrm{P}<0.0001)$ (Fig. $1 \mathrm{H})$.

There was no significant association between PDL1expression in tumor cells and age, gender, tumor size, location, neural invasion, TILs count and CD8 expression.

\section{PD-L1 expression in tumor-infiltrating immune cells}

PD-L1 was expressed in tumor infiltrating immune cells in 24 (28.2\%) cases (Fig. 1I).

In contrast to PD-L1 expression in tumor cells, positive PDL-1 expression in the immune cells within the stroma was significantly associated with less aggressive tumor features such as: low tumor grade $(\mathrm{P}=$ $0.016)$ and absence of LVI $(\mathrm{P}=0.004)$. Ten $(41.7 \%)$ and twenty-two cases (91.7\%) of PD-L1-positive tumors had a high number of tumor infiltrating lymphocytes $(\mathrm{P}<0.0001)$ and high $\mathrm{CD} 8$-positive tumor infiltrating $\mathrm{T}$ cells $(\mathrm{P}=0.002)$ respectively. However, and similar to PD-L1 expression in tumor cells, no association has been detected between PD-L1 expression in tumor-infiltrating immune cells and age, gender, tumor size, location, the presence of lymph node metastases, and perineural invasion (Table 2).

\section{Correlation of CD8-positive $T$ cells with Clinicopathologic Variables}

Clinicopathological associations of CD8+ T-cells are summarized in (Table 3).

Seventeen (20\%) and 68 (80\%) cases contained high and low intratumoral CD8-positive $\mathrm{T}$ cell number, respectively (Figs. 2A and B). Peritumoral CD8positive $\mathrm{T}$ cell was high in 28 patients $(32.9 \%)$ and low in 57 patients $(67.1 \%)$ (Fig. 2C).

High intratumoral CD8-positive $\mathrm{T}$ cells was significantly associated with absence of lymph node involvement $(\mathrm{P}=0.013)$, negative LVI $(\mathrm{P}=0.035)$, high TILs count $(\mathrm{P}=0.016)$, positive $\mathrm{PDL}-1$ expression in the immune cells $(\mathrm{P}=0.002)$, and more frequent absence of distant metastasis, however, no significant association has been detected $(\mathrm{P}=0.076)$. Peritumoral CD8-positive T cell, though, didn't show any significant association with any variable.

Table 1. Clinicopathological characteristics

\begin{tabular}{|c|c|c|c|}
\hline \multicolumn{2}{|c|}{ Clinicopathological variables } & $\mathrm{N}$ & $\%$ \\
\hline \multirow[t]{2}{*}{ Age } & $<60$ & 32 & $37.6 \%$ \\
\hline & $>60$ & 53 & $62.4 \%$ \\
\hline \multirow[t]{2}{*}{ Sex } & Male & 46 & $54.1 \%$ \\
\hline & Female & 39 & $45.9 \%$ \\
\hline \multirow[t]{2}{*}{ Tumor size } & $<5 \mathrm{~cm}$ & 54 & $63.5 \%$ \\
\hline & $>5 \mathrm{~cm}$ & 31 & $36.5 \%$ \\
\hline \multirow[t]{2}{*}{ Site } & $\begin{array}{c}\text { Proximal } \\
\text { colon }\end{array}$ & 43 & $50.6 \%$ \\
\hline & Distal/rectum & 42 & $49.4 \%$ \\
\hline \multirow[t]{3}{*}{ Tumor grade } & Grade 1 & 11 & $12.9 \%$ \\
\hline & Grade 2 & 51 & $60.0 \%$ \\
\hline & Grade 3 & 23 & $27.1 \%$ \\
\hline \multirow[t]{2}{*}{ pT stage } & T1-T2 & 14 & $16.5 \%$ \\
\hline & T3-T4 & 71 & $83.5 \%$ \\
\hline \multirow[t]{2}{*}{ pN stage } & Negative & 66 & $77.6 \%$ \\
\hline & Positive & 19 & $22.4 \%$ \\
\hline \multirow[t]{2}{*}{ Metastasis } & Absent & 74 & $87.1 \%$ \\
\hline & Present & 11 & $12.9 \%$ \\
\hline \multirow{2}{*}{$\begin{array}{l}\text { Medullary } \\
\text { histology }\end{array}$} & Absent & 76 & $89.4 \%$ \\
\hline & Present & 9 & $10.6 \%$ \\
\hline \multirow{2}{*}{$\begin{array}{l}\text { Mucinous } \\
\text { histology }\end{array}$} & Absent & 78 & $91.8 \%$ \\
\hline & Present & 7 & $8.2 \%$ \\
\hline \multirow[t]{2}{*}{ LVI } & Absent & 63 & $74.1 \%$ \\
\hline & Present & 22 & $25.9 \%$ \\
\hline \multirow{2}{*}{$\begin{array}{l}\text { Peri-neural } \\
\text { invasion }\end{array}$} & Absent & 72 & $84.7 \%$ \\
\hline & Present & 13 & $15.3 \%$ \\
\hline \multirow[t]{2}{*}{ TIL } & Low & 56 & $65.9 \%$ \\
\hline & High & 29 & $34.1 \%$ \\
\hline \multirow[t]{2}{*}{ Intra CD8 } & Low & 68 & $80.0 \%$ \\
\hline & High & 17 & $20.0 \%$ \\
\hline \multirow[t]{2}{*}{ Peri CD8 } & Low & 57 & $67.1 \%$ \\
\hline & High & 28 & $32.9 \%$ \\
\hline \multirow[t]{2}{*}{ PD-L1 TC } & Negative & 74 & $87.1 \%$ \\
\hline & Positive & 11 & $12.9 \%$ \\
\hline \multirow[t]{2}{*}{ PD-L1 TIMC } & Negative & 61 & $71.8 \%$ \\
\hline & Positive & 24 & $28.2 \%$ \\
\hline
\end{tabular}

Abbreviations: LVI, lymphovascular invasion; TILs, tumor infiltrating lymphocytes; PD-L1, programmed cell death ligand 1; TC, tumor cells; TIMC, tumor infiltrating immune cells. 
Table 2. Correlation of PD-L1 expression with clinicopathological variables

\begin{tabular}{|c|c|c|c|c|c|c|c|c|c|c|c|}
\hline \multirow{2}{*}{\multicolumn{2}{|c|}{ Clinicopathological variables }} & \multicolumn{4}{|c|}{ PD-L1_tumor } & \multirow[t]{3}{*}{$\mathrm{P}$-value } & \multicolumn{4}{|c|}{ PD-L1_lymph } & \multirow[t]{3}{*}{ P-value } \\
\hline & & \multicolumn{2}{|c|}{ negative } & \multicolumn{2}{|c|}{ positive } & & \multicolumn{2}{|c|}{ negative } & \multicolumn{2}{|c|}{ positive } & \\
\hline & & $\mathrm{N}$ & $\%$ & $\mathrm{~N}$ & $\%$ & & $\mathrm{~N}$ & $\%$ & $\mathrm{~N}$ & $\%$ & \\
\hline \multirow[t]{2}{*}{ Age } & $<60$ & 28 & $37.8 \%$ & 4 & $36.4 \%$ & \multirow[t]{2}{*}{0.925} & 24 & $39.3 \%$ & 8 & $33.3 \%$ & \multirow[t]{2}{*}{0.607} \\
\hline & $>60$ & 46 & $62.2 \%$ & 7 & $63.6 \%$ & & 37 & $60.7 \%$ & 16 & $66.7 \%$ & \\
\hline \multirow[t]{2}{*}{ Sex } & Male & 40 & $54.1 \%$ & 6 & $54.5 \%$ & \multirow[t]{2}{*}{0.976} & 34 & $55.7 \%$ & 12 & $50.0 \%$ & \multirow[t]{2}{*}{0.633} \\
\hline & Female & 34 & $45.9 \%$ & 5 & $45.5 \%$ & & 27 & $44.3 \%$ & 12 & $50.0 \%$ & \\
\hline \multirow[t]{2}{*}{ Tumor size } & $<5 \mathrm{~cm}$ & 46 & $62.2 \%$ & 8 & $72.7 \%$ & \multirow[t]{2}{*}{0.497} & 38 & $62.3 \%$ & 16 & $66.7 \%$ & \multirow[t]{2}{*}{0.706} \\
\hline & $>5 \mathrm{~cm}$ & 28 & $37.8 \%$ & 3 & $27.3 \%$ & & 23 & $37.7 \%$ & 8 & $33.3 \%$ & \\
\hline \multirow[t]{2}{*}{ Site } & Proximal colon & 38 & $51.4 \%$ & 5 & $45.5 \%$ & \multirow[t]{2}{*}{0.715} & 32 & $52.5 \%$ & 11 & $45.8 \%$ & \multirow[t]{2}{*}{0.582} \\
\hline & Distal/rectum & 36 & $48.6 \%$ & 6 & $54.5 \%$ & & 29 & $47.5 \%$ & 13 & $54.2 \%$ & \\
\hline \multirow[t]{3}{*}{ Tumor grade } & Grade 1 & 11 & $14.9 \%$ & 0 & $0.0 \%$ & \multirow[t]{3}{*}{$0.001 *$} & 4 & $6.6 \%$ & 7 & $29.2 \%$ & \multirow[t]{3}{*}{$0.016^{*}$} \\
\hline & Grade 2 & 48 & $64.9 \%$ & 3 & $27.3 \%$ & & 38 & $62.3 \%$ & 13 & $54.2 \%$ & \\
\hline & Grade 3 & 15 & $20.3 \%$ & 8 & $72.7 \%$ & & 19 & $31.1 \%$ & 4 & $16.7 \%$ & \\
\hline \multirow[t]{2}{*}{ pT stage } & T1-T2 & 12 & $16.2 \%$ & 2 & $18.2 \%$ & \multirow[t]{2}{*}{0.87} & 12 & $19.7 \%$ & 2 & $8.3 \%$ & 0.205 \\
\hline & T3-T4 & 62 & $83.8 \%$ & 9 & $81.8 \%$ & & 49 & $80.3 \%$ & 22 & $91.7 \%$ & \\
\hline pN stage & Negative & 61 & $82.4 \%$ & 5 & $45.5 \%$ & $0.006^{*}$ & 45 & $73.8 \%$ & 21 & $87.5 \%$ & 0.171 \\
\hline & Positive & 13 & $17.6 \%$ & 6 & $54.5 \%$ & & 16 & $26.2 \%$ & 3 & $12.5 \%$ & \\
\hline Metastasis & Absent & 65 & $87.8 \%$ & 9 & $81.8 \%$ & 0.579 & 51 & $83.6 \%$ & 23 & $95.8 \%$ & 0.131 \\
\hline & Present & 9 & $12.2 \%$ & 2 & $18.2 \%$ & & 10 & $16.4 \%$ & 1 & $4.2 \%$ & \\
\hline Medullary & Absent & 66 & $89.2 \%$ & 10 & $90.9 \%$ & 0.863 & 53 & $86.9 \%$ & 23 & $95.8 \%$ & 0.227 \\
\hline histology & Present & 8 & $10.8 \%$ & 1 & $9.1 \%$ & & 8 & $13.1 \%$ & 1 & $4.2 \%$ & \\
\hline Mucinous & Absent & 67 & $90.5 \%$ & 11 & $100.0 \%$ & 0.287 & 56 & $91.8 \%$ & 22 & $91.7 \%$ & 0.984 \\
\hline histology & Present & 7 & $9.5 \%$ & 0 & $0.0 \%$ & & 5 & $8.2 \%$ & 2 & $8.3 \%$ & \\
\hline LVI & Absent & 62 & $83.8 \%$ & 1 & $9.1 \%$ & $<0.0001$ & 40 & $65.6 \%$ & 23 & $95.8 \%$ & $0.004 *$ \\
\hline & Present & 12 & $16.2 \%$ & 10 & $90.9 \%$ & & 21 & $34.4 \%$ & 1 & $4.2 \%$ & \\
\hline Peri-neural & Absent & 62 & $83.8 \%$ & 10 & $90.9 \%$ & 0.54 & 52 & $85.2 \%$ & 20 & $83.3 \%$ & 0.825 \\
\hline invasion & Present & 12 & $16.2 \%$ & 1 & $9.1 \%$ & & 9 & $14.8 \%$ & 4 & $16.7 \%$ & \\
\hline TIL & Low & 48 & $64.9 \%$ & 8 & $72.7 \%$ & 0.608 & 54 & $88.5 \%$ & 2 & $8.3 \%$ & $<0.0001$ \\
\hline & High & 26 & $35.1 \%$ & 3 & $27.3 \%$ & & 7 & $11.5 \%$ & 22 & $91.7 \%$ & * \\
\hline Intra CD8 & Low & 58 & $78.4 \%$ & 10 & $90.9 \%$ & 0.332 & 54 & $88.5 \%$ & 14 & $58.3 \%$ & $0.002 *$ \\
\hline & High & 16 & $21.6 \%$ & 1 & $9.1 \%$ & & 7 & $11.5 \%$ & 10 & $41.7 \%$ & \\
\hline Peri CD8 & Low & 48 & $64.9 \%$ & 9 & $81.8 \%$ & 0.264 & 44 & $72.1 \%$ & 13 & $54.2 \%$ & 0.113 \\
\hline & High & 26 & $35.1 \%$ & 2 & $18.2 \%$ & & 17 & $27.9 \%$ & 11 & $45.8 \%$ & \\
\hline
\end{tabular}

*Significant value 
Table 3. Correlations of CD8+ cells (intra- and peri-tumoral) with clinicopathological characteristics

\begin{tabular}{|c|c|c|c|c|c|c|c|c|c|c|c|}
\hline \multirow{3}{*}{\multicolumn{2}{|c|}{ Clinicopathological variables }} & \multicolumn{4}{|c|}{ Intra CD8 } & \multirow{3}{*}{$\begin{array}{c}\mathrm{P}- \\
\text { value }\end{array}$} & \multicolumn{4}{|c|}{ Peri CD8 } & \multirow{3}{*}{$\begin{array}{c}\mathrm{P}- \\
\text { value }\end{array}$} \\
\hline & & \multicolumn{2}{|c|}{ Low } & \multicolumn{2}{|c|}{ High } & & \multicolumn{2}{|c|}{ Low } & \multicolumn{2}{|c|}{ High } & \\
\hline & & $\mathrm{N}$ & $\%$ & $\mathrm{~N}$ & $\%$ & & $\mathrm{~N}$ & $\%$ & $\mathrm{~N}$ & $\%$ & \\
\hline \multirow[t]{2}{*}{ Age } & $<60$ & 23 & $33.8 \%$ & 9 & $52.9 \%$ & \multirow[t]{2}{*}{0.146} & 25 & $43.9 \%$ & 7 & $25.0 \%$ & \multirow[t]{2}{*}{0.092} \\
\hline & $>60$ & 45 & $66.2 \%$ & 8 & $47.1 \%$ & & 32 & $56.1 \%$ & 21 & $75.0 \%$ & \\
\hline \multirow[t]{2}{*}{ Sex } & Male & 34 & $50.0 \%$ & 12 & $70.6 \%$ & \multirow[t]{2}{*}{0.128} & 33 & $57.9 \%$ & 13 & $46.4 \%$ & \multirow[t]{2}{*}{0.319} \\
\hline & Female & 34 & $50.0 \%$ & 5 & $29.4 \%$ & & 24 & $42.1 \%$ & 15 & $53.6 \%$ & \\
\hline \multirow[t]{2}{*}{ Tumor size } & $<5 \mathrm{~cm}$ & 44 & $64.7 \%$ & 10 & $58.8 \%$ & \multirow[t]{2}{*}{0.652} & 34 & $59.6 \%$ & 20 & $71.4 \%$ & \multirow[t]{2}{*}{0.289} \\
\hline & $>5 \mathrm{~cm}$ & 24 & $35.3 \%$ & 7 & $41.2 \%$ & & 23 & $40.4 \%$ & 8 & $28.6 \%$ & \\
\hline \multirow[t]{2}{*}{ Site } & Proximal colon & 36 & $52.9 \%$ & 7 & $41.2 \%$ & \multirow[t]{2}{*}{0.386} & 30 & $52.6 \%$ & 13 & $46.4 \%$ & \multirow[t]{2}{*}{0.591} \\
\hline & Distal/rectum & 32 & $47.1 \%$ & 10 & $58.8 \%$ & & 27 & $47.4 \%$ & 15 & $53.6 \%$ & \\
\hline \multirow[t]{3}{*}{ Tumor grade } & Grade 1 & 9 & $13.2 \%$ & 2 & $11.8 \%$ & \multirow[t]{3}{*}{0.573} & 6 & $10.5 \%$ & 5 & $17.9 \%$ & \multirow[t]{3}{*}{0.392} \\
\hline & Grade 2 & 39 & $57.4 \%$ & 12 & $70.6 \%$ & & 37 & $64.9 \%$ & 14 & $50.0 \%$ & \\
\hline & Grade 3 & 20 & $29.4 \%$ & 3 & $17.6 \%$ & & 14 & $24.6 \%$ & 9 & $32.1 \%$ & \\
\hline \multirow[t]{2}{*}{ pT stage } & T1-T2 & 12 & $17.6 \%$ & 2 & $11.8 \%$ & \multirow[t]{2}{*}{0.559} & 10 & $17.5 \%$ & 4 & $14.3 \%$ & 0.703 \\
\hline & T3-T4 & 56 & $82.4 \%$ & 15 & $88.2 \%$ & & 47 & $82.5 \%$ & 24 & $85.7 \%$ & \\
\hline pN stage & Negative & 49 & $72.1 \%$ & 17 & $100.0 \%$ & $0.013 *$ & 42 & $73.7 \%$ & 24 & $85.7 \%$ & 0.211 \\
\hline & Positive & 19 & $27.9 \%$ & 0 & $0.0 \%$ & & 15 & $26.3 \%$ & 4 & $14.3 \%$ & \\
\hline Metas & Absent & 57 & $83.8 \%$ & 17 & $100.0 \%$ & 0.076 & 48 & $84.2 \%$ & 26 & $92.9 \%$ & 0.264 \\
\hline & $P$ & 11 & $16.2 \%$ & 0 & $0.0 \%$ & & 9 & $15.8 \%$ & 2 & $7.1 \%$ & \\
\hline Medullary & $\mathrm{nt}$ & 61 & $89.7 \%$ & 15 & $88.2 \%$ & 0.86 & 51 & $89.5 \%$ & 25 & $89.3 \%$ & 0.979 \\
\hline histol & Pre & 7 & $10.3 \%$ & 2 & $11.8 \%$ & & 6 & $10.5 \%$ & 3 & $10.7 \%$ & \\
\hline Mucinous & Absent & 63 & $92.6 \%$ & 15 & $88.2 \%$ & 0.554 & 52 & $91.2 \%$ & 26 & $92.9 \%$ & 0.797 \\
\hline histology & Present & 5 & $7.4 \%$ & 2 & $11.8 \%$ & & 5 & $8.8 \%$ & 2 & $7.1 \%$ & \\
\hline LVI & Absent & 47 & $69.1 \%$ & 16 & $94.1 \%$ & $0.035^{*}$ & 42 & $73.7 \%$ & 21 & $75.0 \%$ & 0.896 \\
\hline & Present & 21 & $30.9 \%$ & 1 & $5.9 \%$ & & 15 & $26.3 \%$ & 7 & $25.0 \%$ & \\
\hline Peri-neural & Absent & 57 & $83.8 \%$ & 15 & $88.2 \%$ & 0.651 & 50 & $87.7 \%$ & 22 & $78.6 \%$ & 0.271 \\
\hline & Present & 11 & $16.2 \%$ & 2 & $11.8 \%$ & & 7 & $12.3 \%$ & 6 & $21.4 \%$ & \\
\hline TIL & Low & 49 & $72.1 \%$ & 7 & $41.2 \%$ & $0.016^{*}$ & 39 & $68.4 \%$ & 17 & $60.7 \%$ & 0.481 \\
\hline & High & 19 & $27.9 \%$ & 10 & $58.8 \%$ & & 18 & $31.6 \%$ & 11 & $39.3 \%$ & \\
\hline PD-L1 TC & Negative & 58 & $85.3 \%$ & 16 & $94.1 \%$ & 0.332 & 48 & $84.2 \%$ & 26 & $92.9 \%$ & 0.264 \\
\hline & Positive & 10 & $14.7 \%$ & 1 & $5.9 \%$ & & 9 & $15.8 \%$ & 2 & $7.1 \%$ & \\
\hline PD-L1 TIMC & Negative & 54 & $79.4 \%$ & 7 & $41.2 \%$ & $0.002 *$ & 44 & $77.2 \%$ & 17 & $60.7 \%$ & 0.113 \\
\hline & Positive & 14 & $20.6 \%$ & 10 & $58.8 \%$ & & 13 & $22.8 \%$ & 11 & $39.3 \%$ & \\
\hline
\end{tabular}

*Significant value 

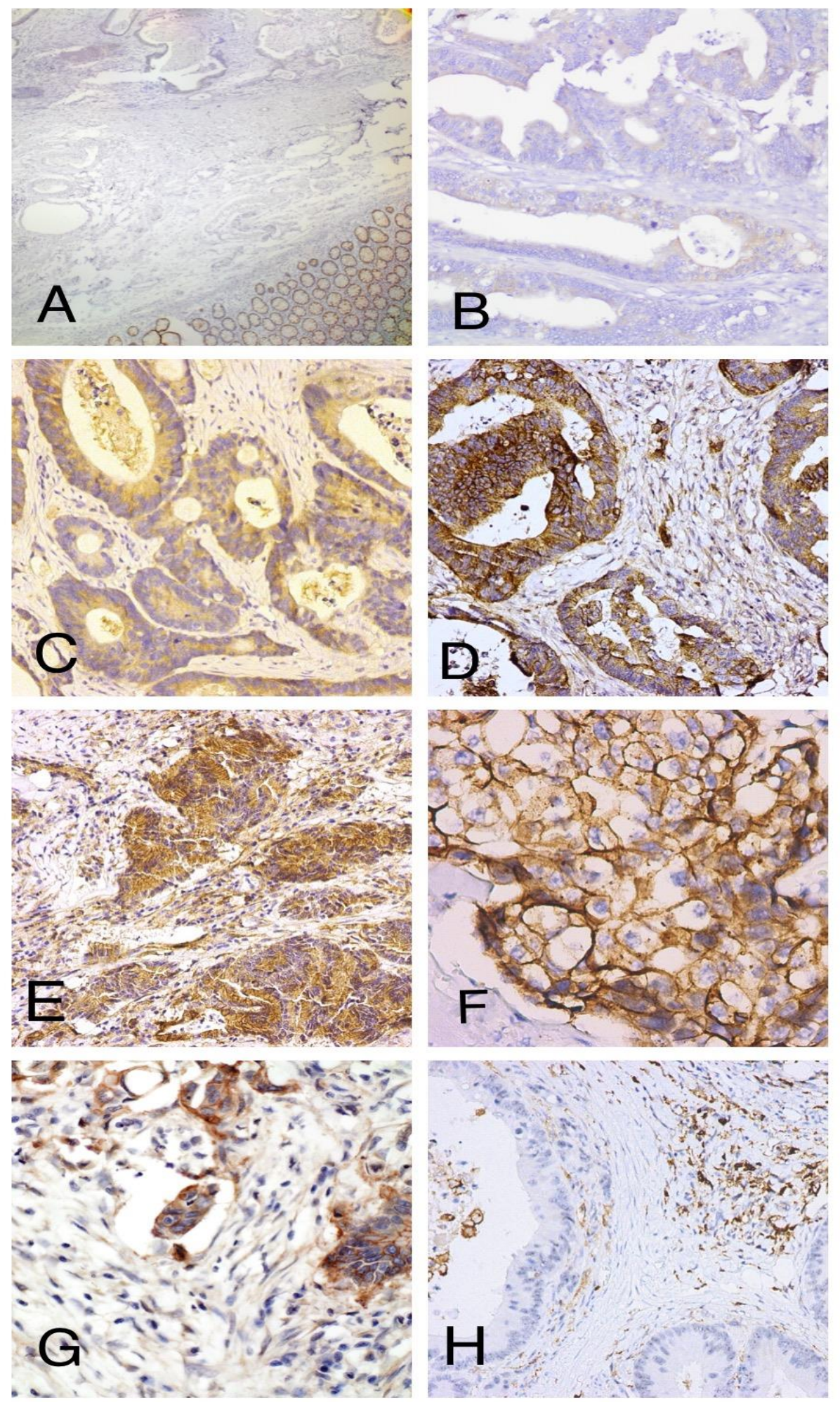

Figure 1: colorectal adenocarcinoma with PD-L1 expression; a) well- differentiated adenocarcinoma with negative PD-L1 expression, note the positive expression in normal gland at the left right corner (IHC x40); b) moderately differentiated adenocarcinomas with weak PD-L1 cytoplasmic expression (IHC x 400); c) moderate PD-L1 cytoplasmic expression (IHC x200); d) strong cytoplasmic and membranous expression (IHC x200), note positive PD-L1 expression in TIMC; e) Poorly-differentiated adenocarcinomas with stong PD-L1 cytoplasmic expression (IHC, x200) ; f) membranousn expression (IHC x400),g) lymphovascular invasion showing strong PD-L1 expression in tumor cells and negative expression in TIMC (IHC x400); i) negative PD-L1 expression.in tumor cells and positive expression in TIMC (IHC x200). 


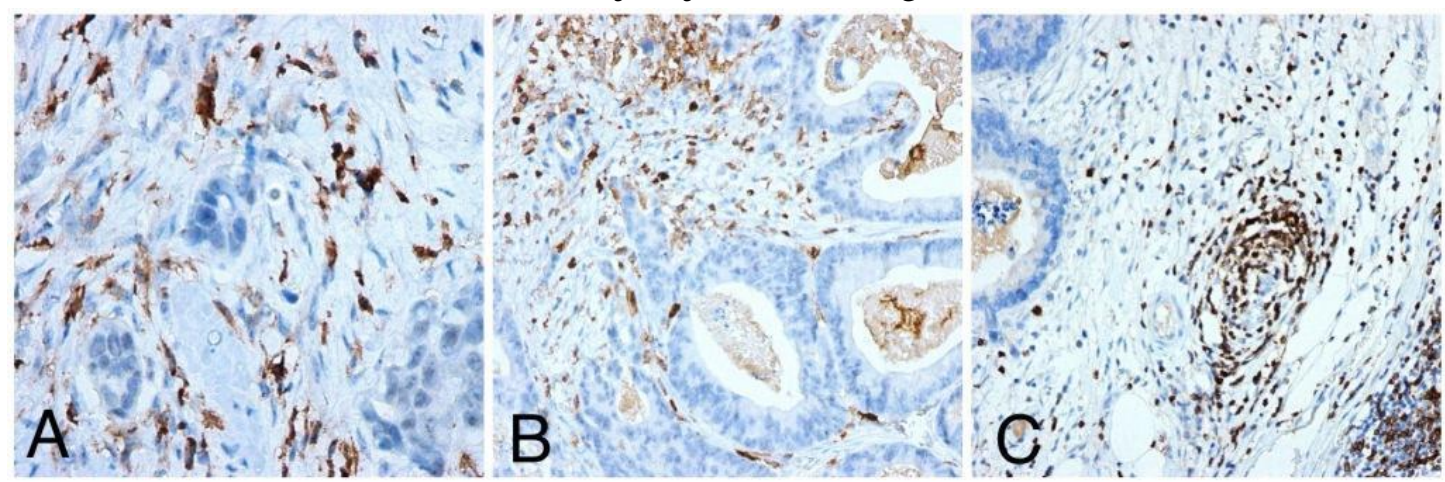

Figure 2: a) high number of intraepithelial CD8+ (IHC x400); b) low number of intraepithelial CD8+ lymphocytes (IHC x200) and c) high number of peritumoral/stromal CD8+ (IHC x200).

\section{DISCUSSION}

Evading the immune surveillance is one of the hallmarks gained during carcinogenesis. Lately, immunotherapeutic agents directed against immunosuppressive proteins, such as PD-L1 have been considered as prospective treatments for cancer and the anti-PD-L1 antibody has revealed encouraging clinical efficacy against several cancer types ${ }^{(4)}$.The PD-1/PDL1 interaction functions as a fundamental regulatory check against an unwarranted immune response to antigens. In the tumor immune microenvironment, the PD-1/PD-L1 signaling axis may induce immune inhibitory/exhaustion signaling to activate $\mathrm{T}$ cells and thus the antitumor immune response was significantly impaired ${ }^{(7)}$. Accumulated evidences indicated that PD1 or PD-L1 antibodies could remove the inhibitory brakes of PD-L1 on cytotoxic T-cells and therefore activate elimination of cancer cells by cytotoxic T-cells. Thus, interrupting the PD1/PD-L1 signal pathway through PD-1/PD-L1 blockades can recover the native antitumor function of $\mathrm{T}$ cells and can facilitate tumor regression. Therefore, targeting the PD-1/PD-L1 immune checkpoint is considered a promising therapeutic strategy for cancer ${ }^{(1,15)}$. However, whether the expression of PD-L1 on tumor cells was associated with the prognosis of CRC remains unclear. Various recent reports had demonstrated that PD-L1 expression on tumor cells was correlated with poor prognosis ${ }^{3,16}$, 17). In contrast, others have shown completely the opposite $^{(14,18)}$. These conflicting results possibly may be due to variations in study designs and methods. In this study, PD-L1 expression in tumor cells was less frequent than TIMC/stromal expression, $12.9 \%$ and $26.2 \%$ of cases respectively on the other hand Nearly similar results have been previously reported ${ }^{(1,10,19)}$. On the other hand, Lee et al. ${ }^{(20)}$ detected PD-L1 expression in tumor cells in only 5\% of cases, while Inaguma $\boldsymbol{e t}$ $\boldsymbol{a l} .{ }^{(5)}$ and Masugi et al. ${ }^{\left({ }^{6}\right)}$ found PD-L1 expression in PD-L1 cells in $30 \%$ and $89 \%$ of cases respectively. More remarkably, a wide range of PD-L1 expression in TIMC/stromal expression have been also reported, ranged from only $2.5 \%$ up to $60.9 \%$ of cases $(6,12,18,21)$. The possible explanations for these contradictory results may be either due to the heterogeneity in PD-L1 antibodies used in different studies or the absence of established consensus regarding PD-L1 scoring, with different cutoff level of PD-L1 positivity. In this study, the cutoff level was set at $>5 \%$. The correlation of PDL1 expression in colon cancer with the different clinicopathological variables is similarly highly debated. As many other investigators reported ${ }^{(10,12,17}$ ${ }^{22)}$, this study demonstrated that PD-L1 positive tumors were more likely to be poorly differentiated, associated with lymph node metastases and lymphovascular invasion. Conversely, PD-L1 expression on TIMC was associated with less aggressive behavior such as low tumor grade and negative LVI. These findings are greatly emphasized the importance of evaluating PD-L1 expression on TC and TIMC separately. Berntsson et al. similarly found that high expression of PD-L1 in immune cells was significantly associated with lower $\mathrm{T}$ and $\mathrm{N}$ stage and better survival, while PD-L1 expression in tumor cells was significantly associated with younger age, high grade, and with right-sided colon cancers ${ }^{(1)}$.On the other hand, Wang et al. reported that there was no significant association between PD-L1 expression in both tumor cells and TILs and various clinicopathologic parameters ${ }^{(19)}$.

On the contrary to our results and unexpectedly, Droeser $\boldsymbol{e t} \boldsymbol{a l}$. found that strong PD-L1 expression in tumor cells was associated with low grade tumors, early stage, absence of lymphovascular invasion, absence of lymph node involvement and a significantly improved 5-year survival ${ }^{(18)}$. Li et $\boldsymbol{a l}$. reported that PD-L1 expression in tumor cells was correlated with less venous invasion, high TILs infiltration, lower T stage, absent metastasis and therefore better prognosis ${ }^{(14)}$.Additionally, in this study, tumors with PD-L1 expression on TIMC were significantly associated with prominent tumor infiltrating lymphocytes and contained a high number of CD8-positive T cells which is consistent with previous results ${ }^{(1,10,18,23,24)}$. On the contrary, Masugi et al. found that the positive expression of PD-L1 in tumor cells was inversely correlated with TILs ${ }^{(6)}$.It is worth noting that peritumoral/stromal CD8 T-cells, in the current study, were noticeably more eminent than intratumoral CD8 T-cells and the existence of high intratumoral CD8 positive T-cells was robustly predictive to the absence of lymph node involvement, negative LVI, high TILs count and positive PDL-1 expression in the immune cells. These data are in keeping with antecedent studies 
(8-10). Koganemaru and his colleagues identified a conceivable explication of this finding which is the possible induction of tumor-specific CD8-positive $\mathrm{T}$ cells secondary to positive expression of PD-L1 on TIMC which is translated to a good prognosis in patients with colon cancer ${ }^{(10)}$.

\section{CONCLUSION}

Findings from the current study suggesed that PDL1expression in tumor cells is considered as a poor prognostic marker for colorectal cancer, whereas PD-L1 expression on TIMC was associated with favorable pathological parameters, therefore, expression of PDL1 can be used as a biomarker to predict the prognosis of colon cancer. Furthermore, it is important to evaluate PD-L1 expression separately on TC and TIMC. Data from this study also showed that high intraumoral CD8 positive T-cells is considered as a good prognostic marker in CRC patients.

\section{REFERENCES}

1. Berntsson J, Eberhard J, Nodin B, Leandersson K, Larsson AH, Jirstrom K (2018): Expression of programmed cell death protein 1 (PD-1) and its ligand PD-L1 in colorectal cancer: relationship with sidedness and prognosis. Oncoimmunology,7(8): 1465165-1465170.

2. Cantero-Cid R, Casas-Martin J, Hernandez-Jimenez E, Cubillos-Zapata C, Varela-Serrano A, Avendano-Ortiz J et al. (2018):PD-L1/PD-1 crosstalk in colorectal cancer: are we targeting the right cells? BMC Cancer,18(1):945-952.

3. Chen XY, Zhang J, Hou LD, Zhang R, Chen W, Fan HN et al. (2018): Upregulation of $\mathrm{PD}-\mathrm{L} 1$ predicts poor prognosis and is associated with miR-191-5p dysregulation in colon adenocarcinoma. Int. J. Immunopathol. Pharmacol., 60:30045644-30045649.

4. Yaghoubi N, Soltani A, Ghazvini K, Hassanian SM and Hashemy SI (2019): PD-1/ PD-L1 blockade as a novel treatment for colorectal cancer. Biomed. Pharmacother.,110:312-320.

5. Inaguma S, Lasota J, Wang Z, Felisiak-Golabek A, Ikeda H, Miettinen M (2017): Clinicopathologic profile, immunophenotype, and genotype of CD274 (PD-L1)-positive colorectal carcinomas. Mod. Pathol., 30(2):278-285.

6. Masugi Y, Nishihara R, Yang J, Mima K, da Silva A, Shi Y et al. (2017): Tumour CD274 (PD-L1) expression and T cells in colorectal cancer. Gut, 66(8):1463-1473.

7. Dosset M, Vargas TR, Lagrange A, Boidot R, Vegran F, Roussey A et al. (2018):PD-1/PD-L1 pathway: an adaptive immune resistance mechanism to immunogenic chemotherapy in colorectal cancer. Oncoimmunology, 7(6):14339811433998.

8. Teng F, Meng X, Kong L, Mu D, Zhu H, Liu S et al. (2015):Tumor-infiltrating lymphocytes, forkhead box P3, programmed death ligand-1, and cytotoxic $\mathrm{T}$ lymphocyteassociated antigen- 4 expressions before and after neoadjuvant chemoradiation in rectal cancer. Transl. Res., 166(6):721-732 .

9. Koelzer VH, Lugli A, Dawson H, Hadrich M, Berger MD, Borner M et al. (2014): CD8/CD45RO T-cell infiltration in endoscopic biopsies of colorectal cancer predicts nodal metastasis and survival. Journal of Translational Medicine, $12: 81-96$
10. Koganemaru S, Inoshita N, Miura Y, Miyama Y, Fukui Y, Ozaki Y et al. (2017): Prognostic value of programmed deathligand 1 expression in patients with stage III colorectal cancer. Cancer Sci., 108(5):853-8.

11. Lee KS, Kim BH, Oh HK, Kim DW, Kang SB, Kim H et al. (2018): Programmed cell death ligand-1 protein expression and CD274/PD-L1 gene amplification in colorectal cancer: Implications for prognosis. Cancer Sci., 109(9):2957-2969.

12. Wyss J, Dislich B, Koelzer VH, Galvan JA, Dawson H, Hadrich M, et al. (2018): Stromal PD-1/PD-L1 Expression Predicts Outcome in Colon Cancer Patients. Clin. Colorectal. Cancer, 18 (1): 20-38.

13. Amin M ES, Greene F et al. (2017): AJCC Cancer Staging Manual. $8^{\text {th }}$ ed. Springer.New York.

14. Li Y, Liang L, Dai W, Cai G, Xu Y, Li X et al. (2016): Prognostic impact of programed cell death-1 (PD-1) and PDligand 1 (PD-L1) expression in cancer cells and tumor infiltrating lymphocytes in colorectal cancer. Mol. Cancer,15(1): 55-63.

15. Kim JH, Park HE, Cho NY, Lee HS and Kang GH (2016): Characterisation of PD-L1-positive subsets of microsatelliteunstable colorectal cancers. Br. J. Cancer, 115(4):490-496.

16. Song M, Chen D, Lu B, Wang C, Zhang J, Huang L et al. (2013): PTEN loss increases PD-L1 protein expression and affects the correlation between PD-L1 expression and clinical parameters in colorectal cancer. PLoS One, 8(6):65821-6589.

17. Zhu H, Qin H, Huang Z, Li S, Zhu X, He J et al. (2015): Clinical significance of programmed death ligand-1 (PD-L1) in colorectal serrated adenocarcinoma. Int .J. Clin. Exp.Pathol., 8(8):9351-9359.

18. Droeser RA, Hirt C, Viehl CT, Frey DM, Nebiker C, Huber X, et al. (2013): Clinical impact of programmed cell death ligand 1 expression in colorectal cancer. Eur. J .Cancer, 49(9):2233-2242.

19. Wang L, Ren F, Wang Q, Baldridge LA, Monn MF, Fisher KW et al. (2016): Significance of Programmed Death Ligand 1 (PD-L1) Immunohistochemical Expression in Colorectal Cancer. Mo. Diag. Ther., 20(2):175-181.

20. Lee LH, Cavalcanti MS, Segal NH, Hechtman JF, Weiser MR, Smith JJ et al. (2016): Patterns and prognostic relevance of PD-1 and PD-L1 expression in colorectal carcinoma. Mod. Pathol., 29(11):1433-1442.

21. Taube JM, Klein A, Brahmer JR, Xu H, Pan X, Kim JH et al. (2014): Association of PD-1, PD-1 ligands, and other features of the tumor immune microenvironment with response to anti-PD-1 therapy. Clinical cancer research : an official journal of the American Association for Cancer Research, 20(19):5064-5074.

22. Saigusa S, Toiyama Y, Tanaka K, Inoue Y, Mori K, Ide S et al. (2016): Implication of programmed cell death ligand 1 expression in tumor recurrence and prognosis in rectal cancer with neoadjuvant chemoradiotherapy. Int.J. Clin .Oncol., 21(5):946-952.

23. Rosenbaum MW, Bledsoe JR, Morales-Oyarvide V, Huynh TG and Mino-Kenudson M (2016): PD-L1 expression in colorectal cancer is associated with microsatellite instability, BRAF mutation, medullary morphology and cytotoxic tumor-infiltrating lymphocytes. Mod. Pathol., 29(9):1104-1112.

24. Lee KS, Kwak Y, Ahn S, Shin E, Oh HK, Kim DW et al. (2017): Prognostic implication of CD274 (PD-L1) protein expression in tumor-infiltrating immune cells for microsatellite unstable and stable colorectal cancer. Cancer Immunol Immunother., 66(7):927-939. 